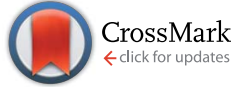

Cite this: J. Mater. Chem. A, 2016, 4, 14234

Received 23rd June 2016 Accepted 17th August 2016

DOI: $10.1039 / c 6 t a 05303 c$

www.rsc.org/MaterialsA

\title{
High performance all-small-molecule solar cells: engineering the nanomorphology via processing additives $\uparrow$
}

\author{
Jie Min, ${ }^{\text {a }}$ Oh Kyu Kwon, ${ }^{\mathrm{b}}$ Chaohua Cui, ${ }^{\text {c }}$ Jung-Hwa Park, ${ }^{\mathrm{b}}$ Yue Wu, \\ Soo Young Park, ${ }^{\text {b }}$ Yongfang $\mathrm{Li}^{\mathrm{c}}$ and Christoph J. Brabec ${ }^{\mathrm{ad}}$
}

\begin{abstract}
The use of small volumes of solvent additives (SAs) or little amounts of non-volatile additives is a processing approach that has been implemented in many high/record performing bulk heterojunction (BHJ) organic solar cells (OSCs). Here, the effects of six SA systems and a molecular additive di-2-thienyl-2,1,3benzothiadiazole (DTBT) were studied with respect to the photovoltaic parameters of solutionprocessed all small molecule solar cells (all-SMSCs) based on the BDTT-S-TR:NIDCS-MO system. An effective strategy with binary additives has been employed in this all-SM system, where a small amount, 0.75 vol\% 1,8-diiodooctane (DIO) and 2 wt\% DTBT were added to the casting solution. This efficient SA approach yielded the highest power conversion efficiency (PCE) of 5.33\%. The relevant additives facilitate phase separation in the $\mathrm{nm}$ domains and improve bulk transport as evidenced by photoluminescence $(\mathrm{PL})$, atomic force microscopy (AFM), X-ray diffraction (XRD) and space charge limited current (SCLC) measurements.
\end{abstract}

\section{Introduction}

Thin-film organic solar cells (OSCs) are under intense study because they offer the possibility of producing low-cost solar cells via solution methods that can enable high throughput fabrication on flexible substrates. ${ }^{1-4}$ Using conventional fullerene derivatives as acceptors, the rational design of both polymer and small molecule donor structures has yielded significant enhancements in power conversion efficiencies (PCEs), now exceeding 10\%., ${ }^{5,6}$ Despite the success of fullerene acceptors, they still suffer from several limitations, which significantly constrain the development of new donor materials; these include limited visible light absorption, synthetic difficulty in electronically modifying fullerenes, high costs of high purity materials, and frequently rather poor thermal stability of blends. ${ }^{7,8}$ Therefore, recent research efforts have been devoted

anstitute of Materials for Electronics and Energy Technology (I-MEET), Friedrich-Alexander-University Erlangen-Nuremberg, Martensstraße 7, 91058 Erlangen, Germany. E-mail: Min.Jie@fau.de

${ }^{b}$ Center for Supramolecular Optoelectronic Materials, Department of Materials Science and Engineering, Seoul National University, Seoul 151-744, South Korea. E-mail: parksy@snu.ac.kr

${ }^{c}$ Laboratory of Advanced Optoelectronic Materials, College of Chemistry, Chemical Engineering and Materials Science, Soochow University, Suzhou 215123, China. E-mail: cuichaohua@suda.edu.cn

${ }^{d}$ Bavarian Center for Applied Energy Research (ZAE Bayern), Haberstraße 2a, 91058 Erlangen, Germany

$\dagger$ Electronic supplementary information (ESI) available. See DOI: $10.1039 / \mathrm{c} 6 \mathrm{ta05303c}$ to exploring and understanding alternative acceptors via electron-deficient subunits to replace fullerene derivatives. Considerable efforts were dedicated to developing nonfullerene acceptors (NFAs) such as conjugated polymer and small molecule acceptors, and the highest PCEs of over 10\% were reported for non-fullerene polymer solar cells (PSCs) in single-junction devices. ${ }^{\mathbf{9}, 10}$

To date, polymers are the dominant electron-donor materials in NFA systems due to their remarkable chemical and physical properties. ${ }^{\mathbf{1 1}}$ Nevertheless, "all small molecule" solar cells (all-SMSCs), which consist of a p-type molecule as the donor and an n-type molecule as the acceptor, have some remarkable advantages over fullerene or non-fullerene based PSCs, including a well-defined molecular structure, good batch reproducibility, easy purification and potentially low cost. ${ }^{\mathbf{1 2 , 1 3}}$ However, the PCEs of all-SMSCs are still lower than those of their polymeric counterparts, and only a few papers reported PCEs over 5\%. ${ }^{14,15}$ There are two main restrictions to obtain high device performance: one is the relatively low electron mobility and electron affinity of n-type semiconducting small molecules; ${ }^{16}$ the other is poor phase separation properties caused by enhanced miscibility and improved intermolecular interactions between the donors and acceptors in the films. ${ }^{17,18}$ Therefore, considerable efforts should be dedicated to the design of new n-type small molecules for extending the pool of acceptor materials and for the development of novel processing strategies facilitating a well-defined morphological evolution for high performance all-SMSCs. 
A number of processing techniques, such as use of mixed solvents and additives and thermal and solvent annealing, have been explored to control the bulk morphology to attain multiple structural requirements. ${ }^{\mathbf{1 4 , 1 5 , 1 8 - 2 3}}$ Recent processing approaches, however, have not always been able to overcome the complexity of separating intrinsic from extrinsic effects. ${ }^{14,15,24,25}$ Solvent additive (SA) processing is a common $\mathrm{BHJ}$ deposition technique during blend film formation with widespread utility in high performance OSCs. ${ }^{26-30}$ This method frequently helped to facilitate phase separation with good domain purity and size for improving charge transportation, suppressing recombination and thus achieving high device performance. ${ }^{18}$ In addition, recent investigations also demonstrated that non-volatile additives allow fine-tuning bulk microstructures to facilitate enhanced charge transport properties. ${ }^{31-33}$ Thus, it is expected that the relevant thin film processing strategies are also helpful for the development of all-SM systems.

In this work, we fabricated all-SMSCs consisting of an alkylthio-thienyl-substituted BDT (benzo[1,2- $\left.b: 4,5-b^{\prime}\right]$ dithiophene) based small molecule donor (BDTT-S-TR) ${ }^{12}$ and a $\left(2 E, 2^{\prime} E\right)-3,3^{\prime}-(2,5-d i m e t h o x y-1,4-p h e n y l e n e) b i s(2-(5-(4-(N-(2-$ ethylhexyl)-1,8-naphthalimide)yl) thiophen-2-yl)acrylonitrile) (NIDCS-MO) acceptor with six SAs and one molecular additive di-2-thienyl-2,1,3-benzothiadiazole (DTBT) (Scheme 1). ${ }^{17}$ The untreated device based on the BDTT-S-TR:NIDCS-MO system had a PCE of approximately $1.0 \%$, but this increased to over $4.5 \%$ when 0.75 vol\% of 1,8 -diiodooctane (DIO) was added. Six SA systems are further investigated with respect to improving the device performance but none of them can be close to the effect of DIO (Table 1). Furthermore, DTBT as a non-volatile additive to reduce donor/acceptor (D/A) mixing was added to DIO. A new processing approach, which is addition of 0.75

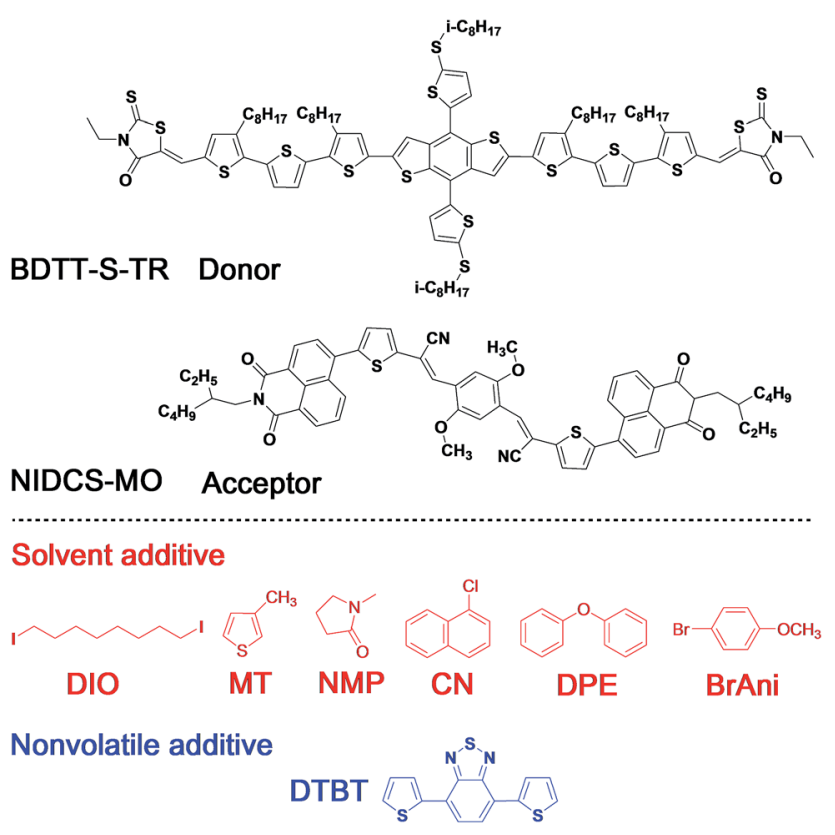

Scheme 1 Molecular structures of BDTT-S-TR and NIDCS-MO as well as different additives used in this system. vol\% DIO and $2 \mathrm{wt} \%$ DTBT into chloroform, allowed further increase of the PCE beyond 5\%. The outcome of this study fosters the discussion on binary additives to control the bulk morphology of solution processed all-SMSCs and other thin film devices.

\section{Results and discussion}

NIDCS-MO has a lowest unoccupied molecular orbital (LUMO) of $-3.66 \mathrm{eV}$ and a highest occupied molecular orbital (HOMO) of $-5.75 \mathrm{eV}$, significantly lower than those of BDTT-S-TR (Fig. S1a, ESI $\dagger$ ). The LUMO and HOMO gaps of $0.54 \mathrm{eV}$ and 0.43 $\mathrm{eV}$, respectively, are large enough to guarantee efficient charge transfer and separation at the D/A interface. In addition, the HOMO and LUMO energy levels of DTBT were estimated by cyclic voltammetry as shown in Fig. S1d $\dagger$ and have been calculated assuming that the HOMO and LUMO energy levels were determined to be -5.49 and $-3.56 \mathrm{eV}$, respectively. They are located between the HOMO and LUMO levels of BDTTS-TR and NIDCS-MO (see Fig. S1a $\dagger$ ). Notably, it suggests that excitons might energetically dissociate at BDTT-S-TR:DTBT, DTBT:NIDCS-MO, and BDTT-S-TR:NIDCS-MO interfaces. UV-Vis absorption spectra of BDTT-S-TR, NIDCS-MO and DTBT in dilute chloroform solutions and in thin films are presented in Fig. S1 $\uparrow$ and the corresponding optical data are collected in Table S1. $\dagger$ The spectra of BDTT-S-TR thin films tend to be significantly red-shifted and possess an obvious shoulder peak at $632 \mathrm{~nm}$, indicating high molecular ordering in the solid state. ${ }^{12}$ No red-shift in the absorption peak is observed for NIDCS-MO films, see Fig. $\mathrm{S} 1 \mathrm{~b}, \uparrow$ which reveals a poor aggregation in the solid state. In addition, the absorption edges of DTBT are at $511 \mathrm{~nm}$ for solutions and at $584 \mathrm{~nm}$ for thin films, corresponding to optical bandgaps of 2.43 and $2.12 \mathrm{eV}$, respectively, which also indicates intermolecular interactions leading to DTBT molecular aggregation in the solid state (see Fig. S1c $\dagger$ ). As a result, the energy levels of these compounds as well as their optical features in the solid state can give a clear conclusion that DTBT molecules work as a sensitizer in this allSM system. This amorphous character of NIDCS-MO directly results in poor charge transport properties of the pristine films. As presented in Fig. S2, $\uparrow$ the hole and electron mobilities of pristine NIDCS-MO films are $6.69 \times 10^{-8}$ and $5.56 \times 10^{-5} \mathrm{~cm}^{2}$ $\mathrm{V}^{-1} \mathrm{~s}^{-1}$, respectively. Note that the conformational changes and the molecular stacking structures of this NIDCS-MO compound could be significantly influenced by thermal annealing treatment, ${ }^{34}$ which have been further demonstrated in a highly efficient all-small molecule system based on NIDCS-MO as the acceptor. ${ }^{17}$

All-SMSCs with a conventional structure of ITO/PEDOT:PSS/ BDTT-S-TR:NIDCS-MO/Ca/Al were fabricated using BDTT-STR:NIDCS-MO blends with different D/A weight ratios and additive contents. The devices based on a D/A weight ratio of $1: 0.8$ exhibit the best photovoltaic performance (Table S2 $\dagger$ ): the open circuit voltage $\left(V_{\mathrm{oc}}\right)$, short circuit current density $\left(J_{\text {sc }}\right)$, fill factor $(\mathrm{FF})$ and power conversion efficiency (PCE) are $1.12 \mathrm{~V}, 3.59 \mathrm{~mA} \mathrm{~cm}{ }^{-2}, 27.1 \%$ and $1.09 \%$, respectively. Six SA systems, including DIO, 3-methylthiophene (MT), $N$-methyl-2- 
Table 1 Average and best device data based on BDTT-S-TR : NIDCS-MO (1:0.8, wt\%) blended films without or with additives in chloroform

\begin{tabular}{|c|c|c|c|c|c|c|c|}
\hline Device & SAs (vol\%) & DTBT (wt\%) & $V_{\mathrm{oc}}(\mathrm{V})$ & $J_{\mathrm{sc}}\left(\mathrm{mA} \mathrm{cm} \mathrm{cm}^{-2}\right)$ & $\mathrm{FF}(\%)$ & $\mathrm{PCE}_{\max }[\%]$ & $\operatorname{PCE}_{\text {ave }}{ }^{a}[\%]$ \\
\hline 1 & - & - & 1.12 & 3.59 & 27.1 & 1.09 & 0.92 \\
\hline 2 & DIO (0.75) & - & 0.97 & 8.29 & 58.1 & 4.67 & 4.50 \\
\hline 4 & $\operatorname{NMP}(0.20)$ & - & 1.03 & 3.86 & 32.7 & 1.30 & 1.16 \\
\hline 5 & $\mathrm{CN}(0.50)$ & - & 1.03 & 6.13 & 42.3 & 2.67 & 2.56 \\
\hline 6 & DPE (0.75) & - & 1.00 & 7.19 & 45.5 & 3.26 & 2.91 \\
\hline 9 & DIO (0.75) & 2 & 0.97 & 9.12 & 60.3 & 5.33 & 5.07 \\
\hline
\end{tabular}

${ }^{a}$ The average PCE value is obtained from six devices.

pyrrolidone (NMP), 1-chloronaphthalene (CN), diphenyl ether (DPE) and 4-bromoanisole (BrAni), have been applied to modify the blend morphologies. As shown in Fig. 1a and Table 1, with the addition of $0.75 \mathrm{vol} \%$ DIO, $J_{\mathrm{sc}}$ increased to $8.29 \mathrm{~mA} \mathrm{~cm}^{-2}$ and the FF increased to $58.1 \%$, which resulted in a PCE of $4.67 \%$. In addition, the other SAs also lead to improved device performance, but at a lower level than that of DIO (Fig. S3, S4† and Table 1).

Since DTBT molecules show significant crystallization behavior in the solid state (see Fig. S1c $\dagger$ ) as well as suitable HOMO and LUMO energy levels, we would expect that DTBT molecules as a non-volatile additive and sensitizer are applied in this all-SM system to further modify the bulk microstructure and enhance light harvesting. The solar cells exhibit the best photovoltaic performance at a DTBT concentration of $3 \mathrm{wt} \%$, which shows a $V_{\mathrm{oc}}$ of $1.00 \mathrm{~V}$, a $J_{\mathrm{sc}}$ of $6.26 \mathrm{~mA} \mathrm{~cm}^{-2}$, a $\mathrm{FF}$ of $36.9 \%$ and a PCE of $2.31 \%$ (Fig. S5 $\dagger$ ). As compared to the initial devices without DTBT molecules, the significantly improved photovoltaic properties of $3 \%$ DTBT based cells are mainly contributed by the improved blend morphology. Furthermore, after the addition of $2 \mathrm{wt} \%$ DTBT together with $0.75 \mathrm{vol} \%$ DIO, the PCE of the all-SM device increased to $5.33 \%$ along with a $V_{\text {oc }}$ of $0.97 \mathrm{~V}$, a $J_{\text {sc }}$ of $9.12 \mathrm{~mA} \mathrm{~cm}^{-2}$ and a FF of $60.3 \%$ (Fig. 1a and Table 1). Note that the former solvent system is now consisting of chloroform and DIO (0.75 vol\%) as the processing solvent, with different weight ratios of DTBT as presented in Fig. S6 $†$. The related $J_{\mathrm{sc}}$ differences have been cross-checked with external quantum efficiency (EQE) measurements, as exhibited in Fig. 1b and S4b. $\uparrow$ The BDTT-S-TR:NIDCS-MO device processed with 0.75 vol\% DIO and $2 \mathrm{wt} \%$ DTBT shows a broad EQE spectrum from $350 \mathrm{~nm}$ to $750 \mathrm{~nm}$ with the maximum of $c a .50 \%$, which is higher than that of the other devices in the whole spectrum. Notably, since the absorption range of DTBT is $c a .400-600 \mathrm{~nm}$ (Fig. S1†), it's difficult to quantify the impact of DTBT on the final device performance.

To better understand the effect of these additives on the bulk microstructures and charge transport properties of these films, optical absorption and photoluminescence (PL) spectra as well as space charge limited current (SCLC) spectroscopy were employed. Among these all-SM films processed with additives including DIO, DTBT and their combination, there are obvious increases of the relative absorption intensity in the region of 450-700 $\mathrm{nm}$ and absorption shoulders in $580 \mathrm{~nm}$ (Fig. 2a), indicating that these additives indeed facilitate molecular ordering in the solid state. The fluorescence intensity of these films decreases significantly (by approximately 90\%) compared to that of pristine BDTT-S-TR and NIDCS-MO films as presented in Fig. 2a. The efficient PL quenching of the untreated films suggests efficient photoinduced charge transport at the D/A interface, resulting from a strongly inter-mixed BDTT-STR:NIDCS-MO microstructure with no significant crystallization or phase separation observable. The enhanced PL intensities of the SA treated films indicate that the phase purity and domain size increase, which is in excellent agreement with the device findings. We thus conclude that DIO indeed facilitates molecular ordering and local phase separation during the active layer
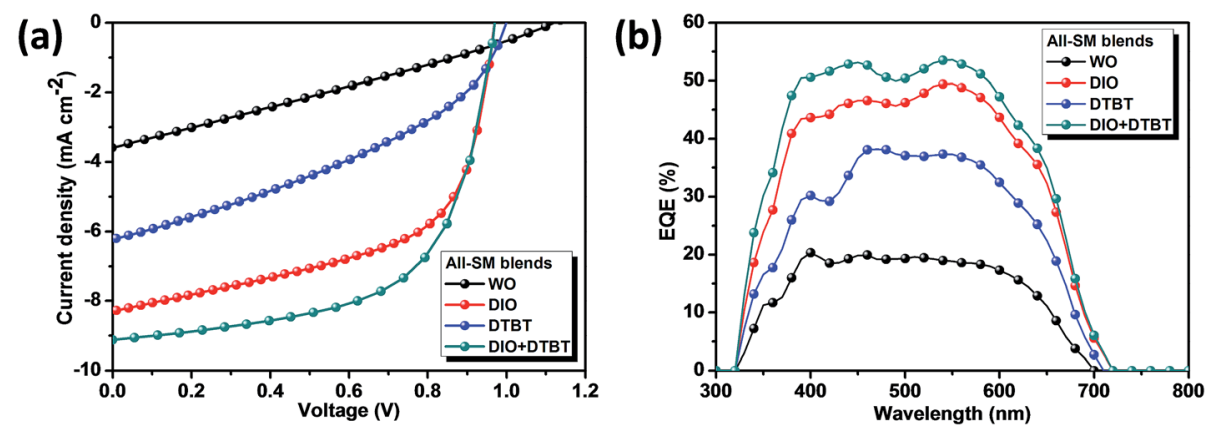

Fig. 1 (a) Current density-voltage ( $J-V$ characteristics and (b) EQE spectra of the OSCs without and with additives under the illumination of an AM 1.5G solar simulator. 

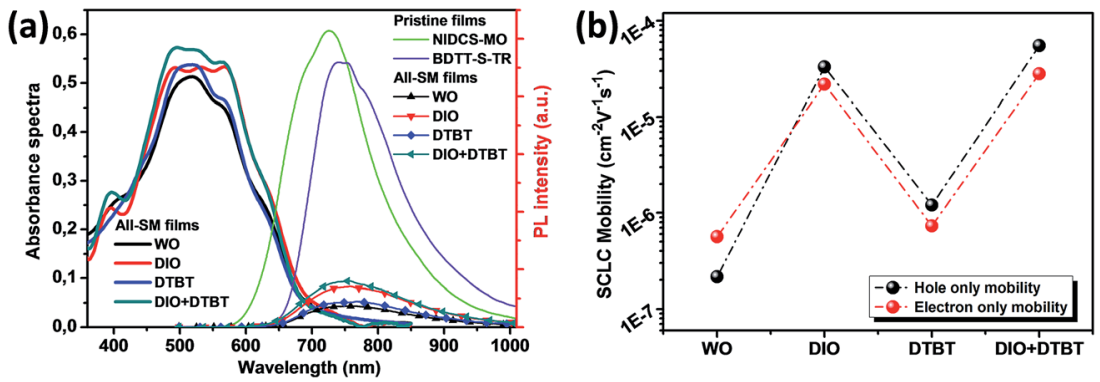

Fig. 2 (a) Left axis: UV-Vis absorption spectra of the BDTT-S-TR:NIDCS-MO films under various processing conditions; right axis: PL spectra of pristine BDTT-S-TR and NIDCS-MO films as well as PL spectra of blended films under different processing conditions. Absorption spectra and PL spectra of the films were measured from the same active layer. (b) Hole and electron only mobilities measured in single carrier diodes obtained from these films. SCLC measurements were carried out on six devices of each type. The lines are guides to the eye; the symbols are the measurement points.

preparation allowing more effective harvesting of solar photons than the films without additives under the same conditions. Further enhanced absorption and PL emission of the DIO + DTBT films indicate the best efficient donor molecular ordering as well as enhanced light harvesting among all SA approach treated all-SM blends.

Then, we determined the hole and electron only mobilities from SCLC measurements (Fig. S7† and Table 2). The hole and electron mobilities of untreated films are $2.16 \times 10^{-7}$ and 5.64 $\times 10^{-7} \mathrm{~cm}^{-2} \mathrm{~V}^{-1} \mathrm{~s}^{-1}$, respectively. The low charge carrier mobility is contributed to the amorphous characteristics of the untreated films. The hole and electron mobilities of DIO treated films are two orders of magnitude higher than those of untreated films. After adding $3 \mathrm{wt} \%$ DTBT into the chloroform host solvent, the electron mobility of deposited films slightly increases from $5.64 \times 10^{-7}$ to $7.32 \times 10^{-7} \mathrm{~cm}^{-2} \mathrm{~V}^{-1} \mathrm{~s}^{-1}$, meanwhile the hole mobility obviously increases from $2.16 \times$ $10^{-7}$ to $1.21 \times 10^{-6} \mathrm{~cm}^{-2} \mathrm{~V}^{-1} \mathrm{~s}^{-1}$. In addition, the hole and electron mobilities of DIO + DTBT films are $5.51 \times 10^{-5}$ and $2.80 \times 10^{-5} \mathrm{~cm}^{-2} \mathrm{~V}^{-1} \mathrm{~s}^{-1}$, which are even slightly larger than those of 0.75 vol\% DIO or $3 \mathrm{wt} \%$ DTBT treated films. The changes between the DIO film and DIO + DTBT film in charge transport properties are not dramatic, but distinct (Fig. 2b), and further data are required to better understand the impact of these additives on the microstructure.

To understand the role of these additives in the BDTT-STR:NIDCS-MO films and to underline the above-mentioned findings from optical and electrical spectroscopy, morphological characterizations, including atomic force microscopy (AFM) and X-ray diffraction (XRD) measurements, were carried out. As shown in Fig. 3, the untreated film shows a smooth surface with a root mean square (RMS) roughness of $1.26 \mathrm{~nm}$. DIO significantly leads to obvious phase separation and subsequent formation of nanoscale domains in the film. Addition of DTBT also shows manifest phase separation; however, the effect is less than that of DIO (Fig. $3 \mathrm{~b}$ and c). The film processed with a combination of DIO and DTBT additives shows again a welldefined phase structure and interconnected domains with tentatively larger donor crystallizes and/or acceptor aggregations. In combination with the optical data (enhanced blend absorption and PL intensities) and the enhanced mobility values, we conclude that DIO in combination with DTBT indeed results in the effective bulk microstructure among the various SA strategies.

To confirm the ordering of BDTT-S-TR upon SA treatments, we finally performed XRD investigations. Fig. 4 plots the specular XRD results of pristine BDTT-S-TR and NIDCS-MO films, while exhibiting the XRD patterns of BHJ films as a function of processing additives. BDTT-S-TR films show high crystallinity, which is tentatively attributed to a strong self-assembly into an ordered lamellar structure. The strong (100) reflection peak at $2 \theta=4.85^{\circ}$ corresponds to a $d_{100^{-}}$spacing value of $18.2 \AA$. In contrast, there is no obvious reflection peak in pristine NIDCSMO films due to their amorphous phase. This result is in good agreement with the above-mentioned optical data (Fig. S1b†). XRD analysis of untreated films also does not exhibit any reflection peaks, underlining the amorphous character of the blend composite. In contrast, weak but obvious reflection peaks

Table 2 Average hole and electron mobilities of all-SM films without and with additives, determined from the SCLC measurements

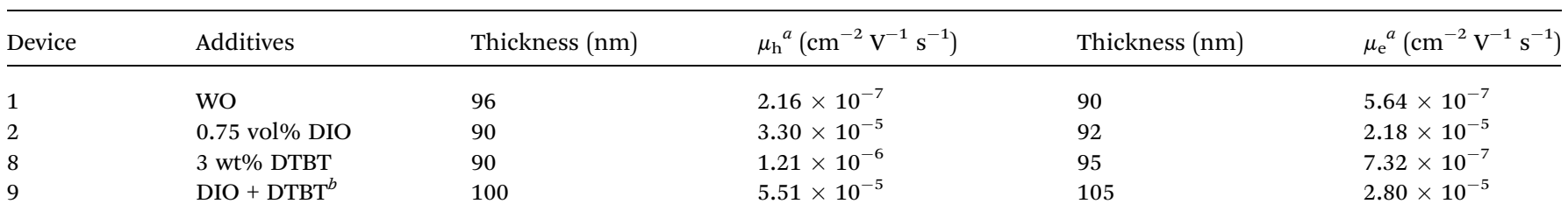

${ }^{a}$ An average value based on six devices with the same thickness. ${ }^{b}$ The volume concentration of DIO is 0.75 vol\% meanwhile the weight concentration of DTBT is $2 \mathrm{wt} \%$ in solutions. 

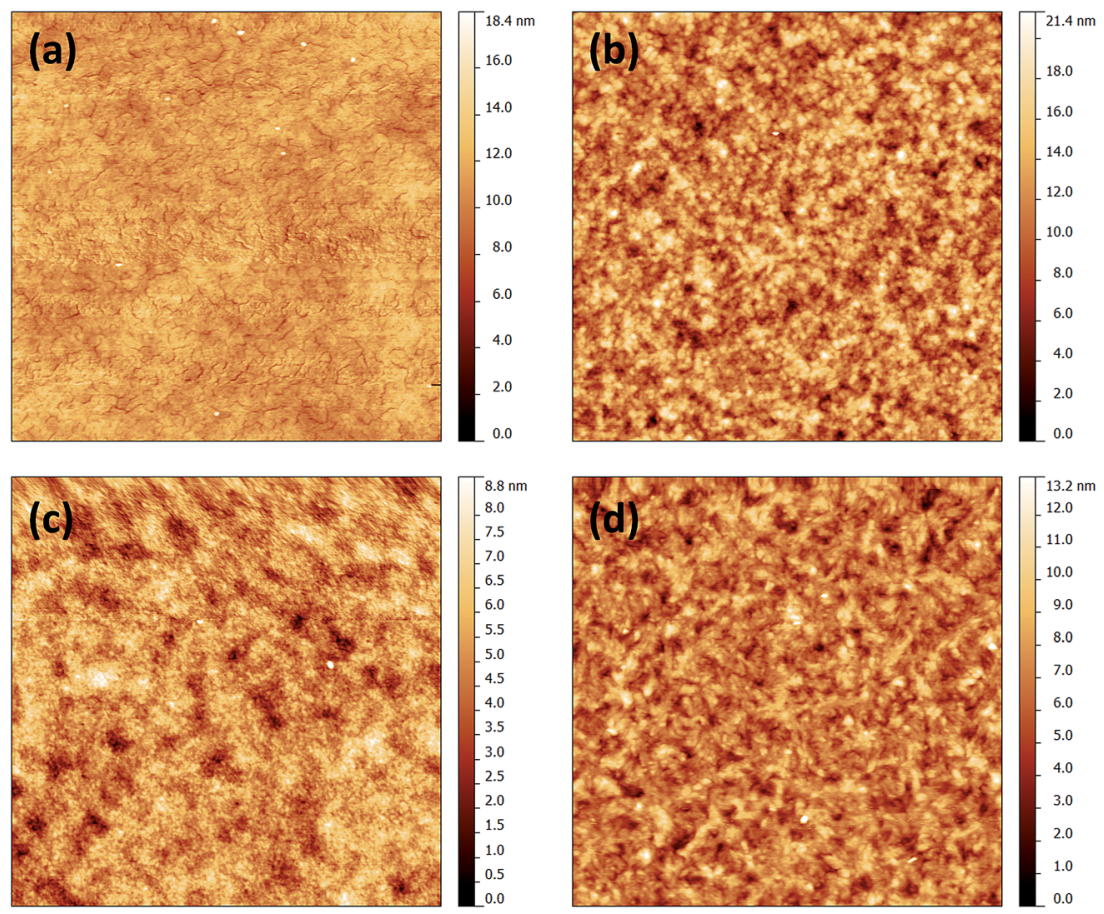

Fig. 3 Tapping-mode AFM surface scans (size: $5 \times 5 \mu \mathrm{m}^{2}$ ) of films based on (a) without any additives (RMS =1.26 nm), (b) with $0.75 \%$ DIO (RMS $=$ $2.79 \mathrm{~nm})$, (c) with $3 \%$ DTBT (RMS $=1.09 \mathrm{~nm}$ ) and (d) with $0.75 \%$ DIO and $2 \%$ DTBT (RMS $=1.55 \mathrm{~nm})$.

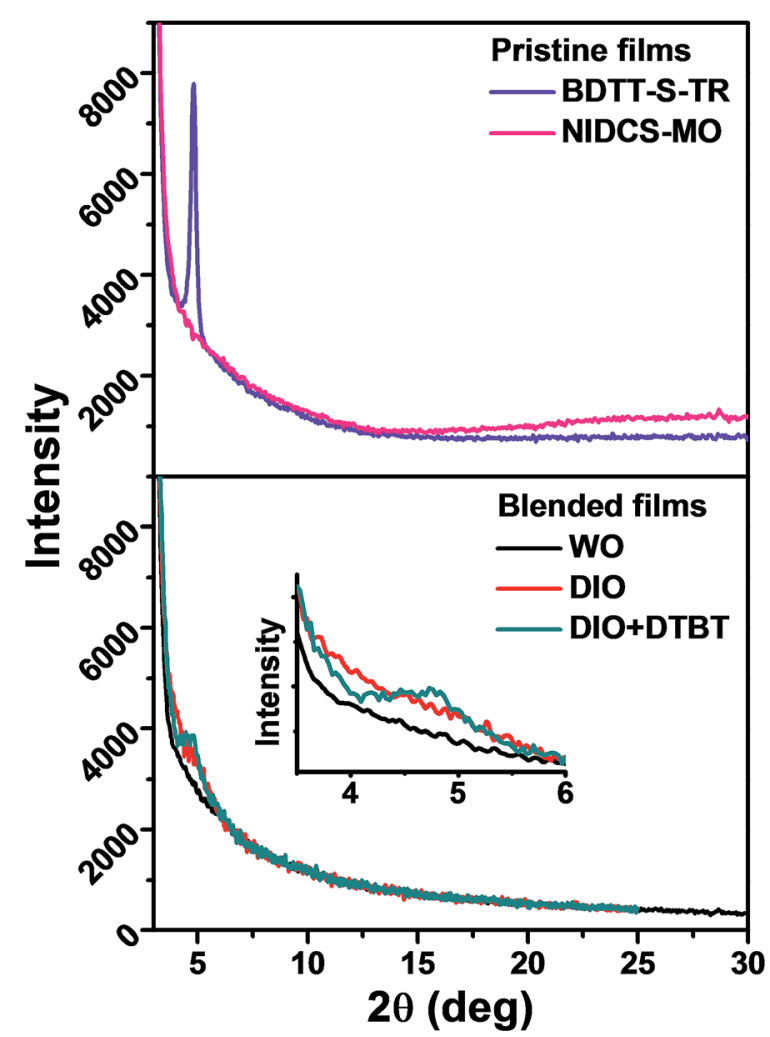

Fig. 4 X-ray diffraction (XRD) measurements. Top: XRD patterns of pristine BDTT-S-TR and NIDCS-MO films; bottom: XRD patterns of BDTT-S-TR : NIDCS-MO (1:0.8, wt\%) films processed under various conditions. are observed for the blend films using DIO or combinations of DIO with DTBT as additives. In addition, only the DTBT treated blend film also exhibits a small reflection peak at $2 \theta=4.85^{\circ}$ (see Fig. S $8 \dagger$ ). Overall, the increase in aggregation and intermolecular interaction of BDTT-S-TR in SA treated films not only demonstrates better charge transport properties but also further explains why charge recombination is suppressed, thus leading to an enhanced $\mathrm{FF}$ and $J_{\mathrm{sc}}$ and an overall enhanced performance. $^{20,35-37}$

\section{Conclusion}

We used six solvent additives and a non-volatile DTBT additive to optimize the BDTT-S-TR:NIDCS-MO blend morphology for synthesizing high performance all-SMSCs. Addition of DIO improved significantly the blend morphology and enhanced the absorption strength as well as the charge transport properties, resulting in a PCE improvement from $1.09 \%$ to $4.67 \%$. Among the six investigated solvent additives, DIO facilitated the most expressed phase separation. When a small amount of DTBT was added to the casting solution, the deposited films exhibited even slightly more favorable phase separation as evidenced by optical and morphological measurements. The binary combination of DIO and DTBT additives finally enhanced the PCE up to $5.33 \%$, mainly due to an improved bulk microstructure, enhanced absorption spectra and increased charge transport properties. Our results demonstrate that using binary additives could be a promising strategy towards high performance all-SMSCs or other organic electronics. 


\section{Experimental section}

Small molecule BDTT-S-TR and DTBT additives were synthesized according to our previously reported procedures. ${ }^{12,38}$ The NIDCS-MO acceptor was synthesized by Prof. Park's group. The detailed synthesis and characterization of NIDCS-MO were reported elsewhere. ${ }^{34}$ Chloroform and solvent additives used in the device fabrication process were purchased from Alfa Aesar or Aldrich Inc.

The all-SMSC devices were fabricated with a structure of Glass/ITO/PEDOT:PSS/BDTT-S-TR:NIDCS-MO/Ca/Al and subsequently processed and characterized in ambient atmosphere. Pre-structured ITO coated glass substrates (as obtained from Osram) were subsequently cleaned in acetone and isopropyl alcohol for $10 \mathrm{~min}$ each. After drying, the substrates were blended with $40 \mathrm{~nm}$ poly(3,4-ethylenedioxythiophene):poly(styrene sulfonate) (PEDOT:PSS. Heraeus, Clevios P VP.Al 4083). A mixture of BDTT-S-TR:NIDCS-MO in chloroform ( $15 \mathrm{mg} \mathrm{mL}^{-1}$ in total) without or with additives was spin-coated on the PEDOT:PSS layer to form a thin film. The thickness of BDTT-S-TR:NIDCS-MO blend films was $c a$. 80-100 $\mathrm{nm}$. Before cathode deposition, the samples were kept in a vacuum chamber at a base pressure below $10^{-7}$ mbar for one hour. Finally, $15 \mathrm{~nm} \mathrm{Ca}$ and $100 \mathrm{~nm} \mathrm{Al}$ were thermally evaporated through shadow masks to form an active area of 10.4 $\mathrm{mm}^{2}$. The current-voltage characteristics of the solar cells were measured under AM 1.5G illumination from an OrielSol1A Solar simulator $\left(100 \mathrm{~mW} \mathrm{~cm}{ }^{-2}\right)$. The light source was calibrated by using a silicon reference cell. The EQE was detected with a Cary 500 Scan UV-vis-NIR Spectrophotometer under mono-chromatic illumination, which was calibrated with a mono-crystalline silicon diode. The thicknesses of the thin films were measured by using a profilometer (Tencor Alpha Step).

Absorption profiles were recorded with a Perkin Elmer Lambda-35 absorption spectrometer from 300 to $850 \mathrm{~nm}$. PL measurements were performed on active layers, which were excited at $445 \mathrm{~nm}$. The PL emission of the films was dispersed by using a 600 lines per mm grating monochromator (HRS-2) and detected by using a silicon $(\mathrm{Si})$ detector through the lock-in technique. The fluorescence spectrum was corrected for the optical density of the sample at the excitation wavelength and for the detection sensitivity of the Si detector. Single carrier devices were fabricated and the dark current-voltage characteristics were measured and analyzed following the space charge limited (SCL) method. ${ }^{12}$ The structure of hole only devices was glass/ITO/PEDOT:PSS/Active layer/MoOx (10 nm)/Ag $(100 \mathrm{~nm})$. For the electron only devices, the structure was glass/ ITO/ZnO/Active layer/Ca $(15 \mathrm{~nm}) / \mathrm{Al}(100 \mathrm{~nm})$. Atomic force microscopy (AFM) measurements were performed with a Nanosurf Easy Scan 2 in the tapping-mode. XRD measurements were conducted using a PANalytical (Empyrean) equipment using $\mathrm{Cu}$ $\mathrm{K} \alpha$ as the excitation source.

Cyclic voltammetry was performed on a Zahner IM6e electrochemical workstation with a three-electrode system in a solution of $0.1 \mathrm{M}\left[\mathrm{Bu}_{4} \mathrm{~N}\right] \mathrm{PF}_{6}$ acetonitrile at a scan rate of $100 \mathrm{mV} \mathrm{s}^{-1}$. A small amount of DTBT was dissolved in the acetonitrile solution, and glassy carbon was used as the working electrode. A Pt wire was used as the counter electrode and $\mathrm{Ag} /$ $\mathrm{Ag}^{+}$was used as the reference electrode. A ferrocene/ferrocenium redox couple was used as the external standard and its redox potential is $0.06 \mathrm{~V} v s$. $\mathrm{Ag} / \mathrm{Ag}^{+}$. The HOMO and LUMO energy levels were calculated from the onset of the oxidation potential of the molecules using the following equations: $\mathrm{HOMO}=-e\left(\varphi_{\mathrm{ox}}+4.74\right)(\mathrm{eV}) ; \mathrm{LUMO}=-e\left(\varphi_{\mathrm{red}}+4.74\right)(\mathrm{eV})$. As shown in Fig. S1d, $\uparrow$ the onset oxidation potentials $\left(\varphi_{\text {ox }}\right)$ and the reduction potentials $\left(\varphi_{\mathrm{red}}\right)$ of DTBT are $0.75 \mathrm{~V}$ and $-1.18 v \mathrm{~s}$. $\mathrm{Ag} /$ $\mathrm{Ag}^{+}$, respectively. Thus, the HOMO and LUMO energy level values of DTBT are $-5.49 \mathrm{eV}$ and $-3.56 \mathrm{eV}$, respectively.

\section{Acknowledgements}

The authors gratefully acknowledge the support of the Cluster of Excellence "Engineering of Advanced Materials" at the University of Erlangen-Nuremberg, which is funded by the German Research Foundation (DFG) within the framework of its "Excellence Initiative". The work at Seoul National University was supported by the National Research Foundation of Korea (NRF) through a grant funded by the Korean government (MSIP; No. 2009-0081571[RIAM0417-20150013]). The work has been also partially funded by the Sonderforschungsbereich 953 "Synthetic Carbon Allotropes". The authors also thank the support of the "Solar Technologies go Hybrid" (SolTech) project and the Energy Campus Nürnberg (EnCN) financed by the Bavarian state government. C. C., Y. W. and Y. L. acknowledge funding from the Jiangsu Provincial Natural Science Foundation (Grant No. BK20150327), Natural Science Foundation of the Jiangsu Higher Education Institutions of China (Grant No. 15KJB430028), and Project Funded by China Postdoctoral Science Foundation (Grant No. 2015M581855).

\section{References}

1 L. Lucera, F. Machui, P. Kubis, H.-D. Schmidt, J. Adams, S. Strohm, T. Ahmad, K. Forberich, H.-J. Egelhaaf and C. J. Brabec, Energy Environ. Sci., 2016, 9, 89.

2 T. Kim, J. H. Kim, T. E. Kang, C. Lee, H. Kang, M. Shin, C. Wang, B. Ma, U. Jeong, T. S. Kim and B. J. Kim, Nat. Commun., 2015, 6, 8547.

3 R. Søndergaard, M. Hösel, D. Angmo, T. T. Larsen-Olsen and F. C. Krebs, Mater. Today, 2012, 15, 36.

4 W. Liu, S. Liu, N. K. Zawacka, T. R. Andersen, P. Cheng, L. Fu, M. Chen, W. Fu, E. Bundgaard, M. Jørgensen, X. Zhan, F. C. Krebs and H. Z. Chen, J. Mater. Chem. A, 2014, 2, 19809. 5 J. Zhao, Y. Li, G. Yang, K. Jiang, H. Lin, H. Ade, W. Ma and H. Yan, Nat. Energy, 2016, 1, 15027.

6 B. Kan, M. Li, Q. Zhang, F. Liu, X. Wan, Y. Wang, W. Ni, G. Long, X. Yang, H. Feng, Y. Zuo, M. Zhang, F. Huang, Y. Cao, T. P. Russell and Y. Chen, J. Am. Chem. Soc., 2015, 137, 3886.

7 N. Zhou, A. S. Dudnik, T. I. Li, E. F. Manley, T. J. Aldrich, P. Guo, H. C. Liao, Z. Chen, L. X. Chen, R. P. Chang, A. Facchetti, M. Olvera de la Cruz and T. J. Marks, J. Am. Chem. Soc., 2016, 138, 1240. 
8 C. B. Nielsen, S. Holliday, H. Y. Chen, S. J. Cryer and I. McCulloch, Acc. Chem. Res., 2015, 48, 2803.

9 W. Zhao, D. Qian, S. Zhang, S. Li, O. Inganas, F. Gao and J. Hou, Adv. Mater., 2016, 28, 4734.

10 S. Holliday, R. S. Ashraf, A. Wadsworth, D. Baran, S. A. Yousaf, C. B. Nielsen, C. H. Tan, S. D. Dimitrov, Z. Shang, N. Gasparini, M. Alamoudi, F. Laquai, C. J. Brabec, A. Salleo, J. R. Durrant and I. McCulloch, Nat. Commun., 2016, 7, 11585.

11 H. Benten, D. Mori, H. Ohkita and S. Ito, J. Mater. Chem. A, 2016, 4, 5340.

12 C. Cui, X. Guo, J. Min, B. Guo, X. Cheng, M. Zhang, C. J. Brabec and Y. F. Li, Adv. Mater., 2015, 27, 7469.

13 J. Min, Y. N. Luponosov, A. Gerl, M. S. Polinskaya, S. M. Peregudova, P. V. Dmitryakov, A. V. Bakirov, M. A. Shcherbina, S. N. Chvalun, S. Grigorian, N. KaushBusies, S. A. Ponomarenko, T. Ameri and C. J. Brabec, Adv. Energy Mater., 2014, 4, 1301234.

14 Y. Lin, J. Wang, T. Li, Y. Wu, C. Wang, L. Han, Y. Yao, W. Ma and X. Zhan, J. Mater. Chem. A, 2016, 4, 1486.

15 O. K. Kwon, M. A. Uddin, J. H. Park, S. K. Park, T. L. Nguyen, H. Y. Woo and S. Y. Park, Adv. Mater., 2016, 28, 910.

16 G. Sauve and R. Fernando, J. Phys. Chem. Lett., 2015, 6, 3770.

17 O. K. Kwon, J. H. Park, D. W. Kim, S. K. Park and S. Y. Park, Adv. Mater., 2015, 27, 1951.

18 A. Sharenko, D. Gehrig, F. Laquai and T.-Q. Nguyen, Chem. Mater., 2014, 26, 4109.

19 S. Holliday, R. S. Ashraf, C. B. Nielsen, M. Kirkus, J. A. Rohr, C. H. Tan, E. Collado-Fregoso, A. C. Knall, J. R. Durrant, J. Nelson and I. McCulloch, J. Am. Chem. Soc., 2015, 137, 898.

20 Y. Huang, E. J. Kramer, A. J. Heeger and G. C. Bazan, Chem. Rev., 2014, 114, 7006.

21 J. Min, X. Jiao, I. Ata, A. Osvet, T. Ameri, P. Bäuerle, H. Ade and C. J. Brabec, Adv. Energy Mater., 2016, 6, 1502579.

22 T. Kassar, N. S. Güldal, M. Berlinghof, T. Ameri, A. Kratzer, B. C. Schroeder, G. L. Destri, A. Hirsch, M. Heeney, I. McCulloch, C. J. Brabec and T. Unruh, Adv. Energy Mater., 2016, 6, 1502025.
23 N. S. Güldal, T. Kassar, M. Berlinghof, T. Ameri, A. Osvet, R. Pacios, G. Li Destri, T. Unruh and C. J. Brabec, J. Mater. Chem. C, 2016, 4, 2178.

24 Z. Li, F. Wu, H. Lv, D. Yang, Z. Chen, X. Zhao and X. Yang, Adv. Mater., 2015, 27, 6999.

25 T. Heumueller, W. R. Mateker, I. T. Sachs-Quintana, K. Vandewal, J. A. Bartelt, T. M. Burke, T. Ameri, C. J. Brabec and M. D. McGehee, Energy Environ. Sci., 2014, 7, 2974.

26 J. D. Lin, O. V. Mikhnenko, T. S. van der Poll, G. C. Bazan and T. Q. Nguyen, Adv. Mater., 2015, 27, 2528.

27 P. Cheng, C. Yan, Y. Li, W. Ma and X. Zhan, Energy Environ. Sci., 2015, 8, 2357.

28 L. A. Perez, J. T. Rogers, M. A. Brady, Y. Sun, G. C. Welch, K. Schmidt, M. F. Toney, H. Jinnai, A. J. Heeger, M. L. Chabinyc, G. C. Bazan and E. J. Kramer, Chem. Mater., 2014, 26, 6531.

29 J. K. Lee, W. L. Ma, C. J. Brabec, J. Yuen, J. S. Moon, J. Y. Kim, K. Lee, G. Bazan and A. J. Heeger, J. Am. Chem. Soc., 2008, 130, 3619.

30 J. Peet, J. Y. Kim, N. E. Coates, W. L. Ma, D. Moses, A. J. Heeger and G. C. Bazan, Nat. Mater., 2007, 6, 497.

31 P. Cheng and X. Zhan, Mater. Horiz., 2015, 2, 462.

32 P. Cheng, L. Ye, X. Zhao, J. Hou, Y. Li and X. Zhan, Energy Environ. Sci., 2014, 7, 1351.

33 L. Zhang, M. Pu, W. Zhou, X. Hu, Y. Zhang, Y. Xie, B. Liu and Y. Chen, J. Phys. Chem. C, 2015, 119, 23310.

34 O. K. Kwon, J.-H. Park, S. K. Park and S. Y. Park, Adv. Energy Mater., 2015, 5, 1400929.

35 C. J. Brabec, M. Heeney, I. McCulloch and J. Nelson, Chem. Soc. Rev., 2011, 40, 1185.

36 J. Min, Y. N. Luponosov, N. Gasparini, M. Richter, A. V. Bakirov, M. A. Shcherbina, S. N. Chvalun, L. Grodd, S. Grigorian, T. Ameri, S. A. Ponomarenko and C. J. Brabec, Adv. Energy Mater., 2015, 5, 1500386.

37 W. Li, K. H. Hendriks, A. Furlan, W. S. Roelofs, S. C. Meskers, M. M. Wienk and R. A. Janssen, Adv. Mater., 2014, 26, 1565. 38 L. Huo, J. Hou, S. Zhang, H. Y. Chen and Y. Yang, Angew. Chem., Int. Ed., 2010, 49, 1500. 